\title{
A Discrete Model of Discriminatory Price Auctions- An Alternative to Menezes-Monteiro*
}

\author{
Hans Haller ${ }^{\dagger}$ and Yvan Lengwiler
}

January 21, 1998

\begin{abstract}
Menezes and Monteiro, Math. Soc. Sci. (1995), show that a multi-unit discriminatory price auction does not have a pure strategy equilibrium unless one imposes some rather special conditions on the demand functions. This non-existence result might indicate a problem either with the underlying auction procedure (as Menezes and Monteiro suggest) or with the modelling approach (as we suggest). We observe that the non-existence problem disappears if bids must come in multiples of smallest units - a realistic feature. Moreover, we show that most of the analysis can be recast in a discrete action model.
\end{abstract}

JEL classification codes. D44, C72.

Keywords. discriminatory price auction, mixed strategies, existence of equilibrium, integer constraints.

\section{Introduction}

Treasuries apply two kinds of formats for auctions of fixed income securities, namely uniform price and discriminatory price auctions. In the first format, price-quantity bids are ordered with respect to price, from top to bottom. The auctioneer accepts quantities up to the amount he is selling and all winners pay the price equivalent to the highest losing bid. In the second format bids are ordered similarly, but each agent pays the amount equal to his bid.

At present, discriminatory auctions for bonds are more often used than uniform auctions, but some countries have been experimenting with the auction format. The United States, for instance, used to apply only discriminatory price auctions, but has recently started to issue some

* This paper was written while Lengwiler was on leave at the Board of Governors of the Federal Reserve System. The views expressed are not necessarily those of the Board of Governors of the Federal Reserve System or of the Swiss National Bank. Nor are they necessarily those of Virginia Polytechnic Institute and State University.

$\dagger$ Virginia Polytechnic Institute and State University.

$\ddagger$ Swiss National Bank and Board of Governors of the Federal Reserve System.

Please address correspondence to Hans Haller, Department of Economics, Virginia Polytechnic Institute, Blacksburg, VA 24061-0316, USA. Phone: (540) 231-7591. email: haller@vt.edu 
bonds via uniform price auctions (Nyborg and Sundaresan, 1996). The Bundesbank switched from uniform price auctions to discriminatory price auctions for their repos (Nautz, 1995).

Much of the debate about how to sell Treasury bills is centered around the question, which of these auction formats yields greater revenues for the Treasury (e.g. Friedman, 1960, Chari and Weber, 1992). In order to compare the two formats, the equilibrium outcomes for each format have to be determined. A rather obvious prerequisite for doing this is the existence of an equilibrium in both auction formats under review.

Menezes and Monteiro (1995) (henceforth, MM) have proposed a highly stylized model of discriminatory price auctions that could qualify as a model of Treasury bill auctions. They show that a Nash equilibrium in pure strategies does not exist, unless bidders face demand functions satisfying special restrictions. The non-existence is a consequence of the discontinuity of payoff functions, which is due to the necessary rationing when both bidders propose the same price. MM also conjecture that utilizing mixed strategies would in general not resolve the nonexistence problem. They suggest that there is a severe non-existence problem that could impair the comparison of the two auction formats.

Non-existence might indicate a problem either with the auction procedure the model is meant to capture, or with the model itself. MM seem to imply the former: "[...] we show that the existence of equilibrium may be a problem even when we consider a simple model of Treasury bill auctions [...]" (MM, page 286). In this paper, we suggest that it is rather the specific model and not the underlying auction procedure that causes the problem. Non-existence is an artifact of the MM model where prices and quantities are continuous variables. We assume instead a finite grid from which price-quantity pairs are chosen. Our note demonstrates that most of the MM analysis can be recast in such a discrete action auction model where the nonexistence problem vanishes. Moreover, a discrete action model provides a better description of reality than the continuous action model, since there are minimal increments (smallest units) of prices and quantities in actual auctions. Adoption of a continuous action model can only be justified on the grounds that it is more amenable to analysis. If the continuous version fails in this respect, we may as well discard it.

Our main findings for the discrete action model are, first, that the finite action model has a (possibly mixed) equilibrium [proposition 1]. MM show that both bidders submit the same price in any pure strategy equilibrium of the continuous action model. We show, second, that mixed equilibria of the finite action game have a similar property: The price supports of the equilibrium strategies of both bidders are close to each other [proposition 2]. With regard to the existence of a pure strategy equilibrium, we show, third, that the necessary and sufficient conditions identified by MM for the continuous action model have similar counterparts in the finite action model [proposition 3]. Fourth, we find that pure strategy equilibria of the finite action model can exhibit some excess demand [corollary 2], but excess demand vanishes in the limit as the grid becomes arbitrarily fine [proposition 5]. Fifth, there is an equivalence relationship between the pure strategy equilibria of the continuous action game and $\epsilon$-equilibria of the finite action game [proposition 6].

In the next section 2 we restate the MM model. We construct a finite version of this model in section 3, and consider the mixed extension in section 4. Section 5 deals with properties of pure strategy equilibria. Section 6 considers the limit behavior of pure strategy equilibria as the grid becomes arbitrarily fine. Section 7 succinctly concludes. 


\section{The Menezes-Monteiro model}

Let us first recall the MM model. We follow their notation. There is a maximum quantity $Y$ of a homogeneous commodity that is to be sold in an auction. There are two bidders, $i=1,2$, each having a demand function $D_{i}:[0, \bar{p}] \rightarrow \mathbb{R}_{+}$, assumed to be continuous and strictly decreasing. Moreover, $D_{1}(0)+D_{2}(0)>Y$ and $D_{i}(\bar{p})=0$ for both $i$. The above assumptions imply that there exists a unique $p^{*}>0$ that clears the market, $D_{1}\left(p^{*}\right)+D_{2}\left(p^{*}\right)=Y$. Bidders are allowed to bid a price between 0 and $\bar{p}$ and a corresponding quantity between 0 and $Y$. $(p, x)$ and $(q, y)$ will be used to denote the strategy chosen by bidder 1 and 2 , respectively. If $p>q$, bidder 1 will receive his demanded quantity $x$ and bidder 2 will receive either $y$ or the remaining quantity $Y-x$, whichever is smaller. If $p<q$, an analogous allocation is implemented, with the roles of both bidders reversed. If both bidders bid the same price, $p=q$, there are two cases to consider. If their joint demand does not exceed the offered quantity, $x+y \leq Y$, then both bidders receive their respective demand. Otherwise, the bidders have to be rationed. The rationing is assumed to be proportional to the bids, i.e. bidder 1 receives $x Y /(x+y)$ units and bidder 2 is allocated $y Y /(x+y)$ units. Payoffs $(=$ consumer rents) are thus defined by

$$
\begin{aligned}
& \pi_{1}^{*}(p, x, q, y):=\int_{0}^{Z_{1}^{*}(p, x, q, y)}\left(D_{1}^{-1}(s)-p\right) d s, \\
& \pi_{2}^{*}(p, x, q, y):=\int_{0}^{Z_{2}^{*}(p, x, q, y)}\left(D_{2}^{-1}(s)-q\right) d s,
\end{aligned}
$$

with

$$
\begin{aligned}
& Z_{1}^{*}(p, x, q, y):= \begin{cases}x, & \text { if } p>q, \\
x Y / \max \{Y, x+y\}, & \text { if } p=q, \\
\min \{x, Y-y\}, & \text { if } p<q,\end{cases} \\
& Z_{2}^{*}(p, x, q, y):= \begin{cases}y, & \text { if } q>p, \\
y Y / \max \{Y, x+y\}, & \text { if } q=p, \\
\min \{y, Y-x\}, & \text { if } q<p .\end{cases}
\end{aligned}
$$

MM find a set of necessary and sufficient conditions for a pair of pure strategies to constitute a Nash equilibrium.

Theorem 1 (Menezes-Monteiro). A quadrupel ( $p, x, q, y)$ constitutes a pure strategy equilibrium point of the continuous action game if and only if

(i) $p=q$,

(ii) $D_{1}(p)+D_{2}(p)=Y$,

(iii) $D_{i}(p)=Z_{i}^{*}(p, x, p, y)$,

(iv) $\int_{0}^{Y-y} D_{1}^{-1}(s) d s \leq \pi_{1}^{*}(p, x, p, y)$ and $\int_{0}^{Y-x} D_{2}^{-1}(s) d s \leq \pi_{2}^{*}(p, x, p, y)$.

\section{A finite version}

In the MM model, a bidder's strategy set is $S:=[0, \bar{p}] \times[0, Y]$. This means that a player can choose prices and quantities with any degree of precision. The model is intended to capture 
Treasury bill auctions. According to U.S. Treasury regulations, Treasury bills, notes, and bonds may be purchased in multiples of $\$ 1000$.- nominal value, and the submitted prices must be equivalent to yields that can be expressed as multiples of half basis points (U.S. Treasury, 1997). So bidders are confronted with "smallest units." To model this, pick some integers $\bar{n} \geq 2$ and $\bar{m} \geq 2$, and let $\alpha:=\bar{p} / \bar{n}$ and $\beta:=Y / \bar{m}$. Then the set

$$
\mathcal{S}:=\underbrace{\{n \alpha: n \in\{1, \ldots, \bar{n}\}\}}_{=: \mathcal{P}} \times \underbrace{\{m \beta: m \in\{1, \ldots, \bar{m}\}\}}_{=: \mathcal{Y}}
$$

is a grid on $S$, and this is the new strategy set we will use. We will call the game with this finite strategy space the finite action game, whereas the original MM game will be referred to as the continuous action game.

Note that in the continuous action game, if both bidders propose the same price, and their joint quantity bid, $x+y$, exceeds the available supply, $Y$, then the first bidder receives $Y x /(x+y)$, and the second bidder receives the rest. Yet, these quantities need not be on the quantity grid, which implies that fractions of smallest quantity units are allocated to the bidders. This is not consistent with the very idea of smallest units. We take care of that in the simplest possible way, by assuming that fractions are disposed of. (2) has to be adapted somewhat. Let

$$
\check{z}_{1}(x, y):=\max \{z \in \mathcal{Y}: z \leq Y x /(x+y)\},
$$

and an analogous definition for $\check{z}_{2}(x, y)$. $\check{z}_{1}(x, y)$ is the next point on the quantity grid weakly below $Y x /(x+y)$. The allocation in the finite action game is then defined by

$$
\begin{aligned}
& Z_{1}(p, x, q, y):= \begin{cases}x, & \text { if } p>q, \\
\min \left\{x, \check{z}_{1}(x, y)\right\}, & \text { if } p=q, \\
\min \{x, Y-y\}, & \text { if } p<q,\end{cases} \\
& Z_{2}(p, x, q, y):= \begin{cases}y, & \text { if } q>p, \\
\min \left\{y, \check{z}_{2}(x, y)\right\}, & \text { if } q=p, \\
\min \{y, Y-x\}, & \text { if } q<p,\end{cases}
\end{aligned}
$$

and payoffs $\pi_{1}$ and $\pi_{2}$ are given by (1), with the limits of integration, $Z_{1}^{*}$ and $Z_{2}^{*}$, replaced with the new expressions $Z_{1}$ and $Z_{2}$. We will also need the following modified demand functions:

In case $\beta \leq D_{i}(p) \leq Y$, set

$$
\begin{aligned}
\check{D}_{i}(p) & :=\max \left\{z \in \mathcal{Y}: z \leq D_{i}(p)\right\}, \\
\hat{D}_{i}(p) & :=\min \left\{z \in \mathcal{Y}: z \geq D_{i}(p)\right\},
\end{aligned}
$$

and

$$
\bar{D}_{i}(p):= \begin{cases}\check{D}_{i}(p) & \text { if } \int_{0}^{\breve{D}_{i}(p)}\left(D_{i}^{-1}(s)-p\right) d s \geq \int_{0}^{\hat{D}_{i}(p)}\left(D_{i}^{-1}(s)-p\right) d s \\ \hat{D}_{i}(p) & \text { if } \int_{0}^{\breve{D}_{i}(p)}\left(D_{i}^{-1}(s)-p\right) d s<\int_{0}^{\hat{D}_{i}(p)}\left(D_{i}^{-1}(s)-p\right) d s\end{cases}
$$

In case $D_{i}(p)<\beta$, set $\bar{D}_{i}(p)=\beta$. In case $D_{i}(p)>Y$, set $\bar{D}_{i}(p)=Y$. 
Except for very small or huge demand, $\hat{D}_{i}(p)$ is the point on the quantity grid which is equal or just greater than $D_{i}(p)$. Similarly, $\check{D}_{i}(p)$ is the point on the quantity grid which is equal or just smaller than $D_{i}(p)$. $\bar{D}_{i}(p)$ is either equal to $\hat{D}_{i}(p)$ or $\check{D}_{i}(p)$, depending on which quantity gives the player higher payoff, given that he has to pay price $p$ per unit he receives.

Remark. If the price grid is relatively fine compared to the quantity grid, and the demand schedule is steep enough, then there can be several market clearing prices, forming the set

$$
\mathbb{P}:=\left\{p \in \mathcal{P}: \bar{D}_{1}(p)+\bar{D}_{2}(p)=Y\right\}
$$

Conversely, if the price grid is relatively coarse compared to the quantity grid, and the demand schedule is flat enough, then $\mathbb{P}$ might be empty. Because demand schedules are downward sloping, $\mathbb{P}$ is the intersection of some interval with the price grid. If this interval is larger than $2 \alpha$, then there is more than one market clearing price; if the size of this interval is between $\alpha$ and $2 \alpha$, then there is exactly one market clearing price; if this interval is smaller than $\alpha$, then there is possibly no (or at most one) market clearing price.

We assume that $\bar{D}_{1}(2 \alpha)+\bar{D}_{2}(2 \alpha)>Y$, making an assumption of the continuous action MM model somewhat stronger. Note that, because both demand functions are assumed to be decreasing, this implies $\bar{D}_{1}(\alpha)+\bar{D}_{2}(2 \alpha)>Y, \bar{D}_{1}(2 \alpha)+\bar{D}_{2}(\alpha)>Y$, and $\bar{D}_{1}(\alpha)+\bar{D}_{2}(\alpha)>Y$; a fact that will be useful later.

\section{The mixed extension}

Let $\tilde{\mathcal{S}}$ be the set of density functions over $\mathcal{S}$, so

$$
f \in \tilde{\mathcal{S}}: \Longleftrightarrow f(p, x) \geq 0 \text { and } \sum_{\mathcal{S}} f(p, x)=1 .
$$

Let $f \in \tilde{\mathcal{S}}$ and $g \in \tilde{\mathcal{S}}$ denote the (possibly) mixed strategies chosen by bidder 1 and bidder 2 , respectively. Define then the payoff functions of the mixed extension of the game as follows,

$$
\begin{aligned}
& \tilde{\pi}_{1}(f, g):=\sum_{(p, x) \in \mathcal{S}} \sum_{(q, y) \in \mathcal{S}} \pi_{1}(p, x, q, y) f(p, x) g(q, y), \\
& \tilde{\pi}_{2}(f, g):=\sum_{(p, x) \in \mathcal{S}} \sum_{(q, y) \in \mathcal{S}} \pi_{2}(p, x, q, y) f(p, x) g(q, y),
\end{aligned}
$$

A Nash Equilibrium (NE) is a strategy profile $(f, g)$ such that

$$
f \in \underset{f^{\prime} \in \tilde{\mathcal{S}}}{\arg \max } \tilde{\pi}_{1}\left(f^{\prime}, g\right) \quad \text { and } \quad g \in \underset{g^{\prime} \in \tilde{\mathcal{S}}}{\arg \max } \tilde{\pi}_{2}\left(f, g^{\prime}\right) .
$$

Existence is immediate in the mixed extension of the finite action model.

Proposition 1 (existence). The mixed extension of the finite action game has a Nash equilibrium.

Proof. This is Nash's (1950) Theorem. 
In the continuous action game, both bidders submit the same price in equilibrium (MM's item $(i)$ in theorem 1). Equilibria of the finite action game in mixed strategies have a similar property: The supports of the price components of both bidders' equilibrium strategies are close to each other.

Proposition 2 (similar price supports). Given a strategy profile $(f, g)$, let $P$ and $Q$ be the support of the price component of $f$ and $g$, respectively. Formally, $P:=\{p \in \mathcal{P}: \exists x f(p, x)>$ $0\}$, and analogously for $Q$. If $(f, g) \in \mathrm{NE}$, then the Hausdorff-distance of $P$ and $Q$ is at most $\alpha$. In other words, $\forall p \in P \exists q \in Q$ such that $q \in\{p-\alpha, p, p+\alpha\}$ and $\forall q \in Q \exists p \in P$ such that $p \in\{q-\alpha, q, q+\alpha\}$.

Proof. Suppose not. Without loss of generality, assume that there exists $p^{*} \in P$ such that $\left\{p^{*}-\alpha, p^{*}, p^{*}+\alpha\right\} \cap Q=\emptyset$. We will show that this will lead to a contradition to the definition of Nash equilibrium. We distinguish two cases.

Case 1, " $\exists q^{*} \in Q$ s.t. $q^{*}>p^{*}+\alpha$." Then bidder 2 can take away some weight from $q^{*}$ and put it on $p^{*}+\alpha$, thereby increasing his expected payoff, contrary to the best response property of $g$. Specifically, consider

$$
g^{\prime}(q, y):= \begin{cases}g(q, y) & \text { for } q \neq q^{*} \text { and } q \neq p^{*}+\alpha \\ 0 & \text { for } q=q^{*} \\ g\left(q^{*}, y\right)+g\left(p^{*}+\alpha, y\right) & \text { for } q=p^{*}+\alpha\end{cases}
$$

and set $\mathcal{S}^{*}=\left\{(q, y) \in \mathcal{S}: q \neq q^{*}, q \neq p^{*}+\alpha\right\}$. Then

$$
\begin{aligned}
\tilde{\pi}_{2}\left(f, g^{\prime}\right)= & \sum_{(p, x) \in \mathcal{S}} \sum_{(q, y) \in \mathcal{S}^{*}} \pi_{2}(p, x, q, y) f(p, x) g(q, y)+ \\
& \sum_{(p, x) \in \mathcal{S}} \sum_{y \in \mathcal{Y}} \pi_{2}\left(p, x, p^{*}+\alpha, y\right) f(p, x)\left[g\left(q^{*}, y\right)+g\left(p^{*}+\alpha, y\right)\right]
\end{aligned}
$$

whereas

$$
\begin{aligned}
& \tilde{\pi}_{2}(f, g)=\sum_{(p, x) \in \mathcal{S}} \sum_{(q, y) \in \mathcal{S}^{*}} \pi_{2}(p, x, q, y) f(p, x) g(q, y)+ \\
& \sum_{(p, x) \in \mathcal{S}} \sum_{y \in \mathcal{Y}}\left[\pi_{2}\left(p, x, p^{*}+\alpha, y\right) f(p, x) g\left(p^{*}+\alpha, y\right)+\right. \\
& \left.+\pi_{2}\left(p, x, q^{*}, y\right) f(p, x) g\left(q^{*}, y\right)\right] .
\end{aligned}
$$

The first payoff is greater, as can be seen from

$$
\tilde{\pi}_{2}\left(f, g^{\prime}\right)-\tilde{\pi}_{2}(f, g)=\sum_{(p, x) \in \mathcal{S}} \sum_{y \in \mathcal{Y}} f(p, x) g\left(q^{*}, y\right)\left[\pi_{2}\left(p, x, p^{*}+\alpha, y\right)-\pi_{2}\left(p, x, q^{*}, y\right)\right] .
$$

To sign this difference, recall that

$$
\pi_{2}\left(p, x, p^{*}+\alpha, y\right)=\int_{0}^{y}\left(D_{2}^{-1}(s)-\left(p^{*}+\alpha\right)\right) d s,
$$


and

$$
\pi_{2}\left(p, x, q^{*}, y\right)=\int_{0}^{y}\left(D_{2}^{-1}(s)-q^{*}\right) d s .
$$

By assumption, $q^{*}>p^{*}+\alpha$. So $\pi_{2}\left(p, x, p^{*}+\alpha, y\right)>\pi_{2}\left(p, x, q^{*}, y\right)$, and hence $\tilde{\pi}_{2}\left(f, g^{\prime}\right)>\tilde{\pi}_{2}(f, g)$. So $g^{\prime}$ is a better response for bidder 2 against $f$, contradicting the Nash equilibrium property.

Case 2, " $\forall q \in Q q<p^{*}-\alpha$." In other words, $\max Q<p^{*}-\alpha$. In this case, as before, but with reversed roles for both players, bidder 1 can decrease the weight on $\left(p^{*}, x\right)$ and increase the weight on $(\max Q+\alpha, x)$, for all $x$, thereby increasing his expected payoff, given strategy $g$ of bidder 2 .

QED

\section{$5 \quad$ Equilibria in pure strategies}

In this section we provide necessary and sufficient conditions for a pure strategy equilibrium to exist (proposition 3) - a finite game counterpart of MM's theorem. We start by exploiting immediate implications of proposition 2 for Nash equilibria in pure strategies.

Corollary 1 Let $(p, x, q, y) \in \mathcal{P} \times \mathcal{Y} \times \mathcal{P} \times \mathcal{Y}$ be a Nash equilibrium in pure strategies. Then either $p=q$ or $(p, q)=(\alpha, 2 \alpha)$ or $(p, q)=(2 \alpha, \alpha)$.

Proof. Suppose $(p, x, q, y)$ is a Nash equilibrium. As a corollary of proposition 2 we know that $|p-q| \leq \alpha$. There are three possible cases: $p=q, p=q-\alpha$, and $p=q+\alpha$. Suppose $q>2 \alpha$.

We want to show that in these circumstances, no best reply of player 1 will have a price component $p=q-\alpha$. To see this, note first that if $p=q-\alpha$, then bidder 1 will either receive his bid $x$, or the amount not purchased by bidder $2, Y-y$, so $Z_{1}(q-\alpha, x, q, y)=\min \{x, Y-y\}$. Consider then the alternative strategy $\left(\alpha, \bar{D}_{1}(\alpha)\right)$. We have

$$
\begin{aligned}
\pi_{1}(q-\alpha, x, q, y) & =\int_{0}^{\min \{x, Y-y\}}\left(D_{1}^{-1}(s)-(q-\alpha)\right) d s \\
& <\int_{0}^{\min \{x, Y-y\}}\left(D_{1}^{-1}(s)-\alpha\right) d s \\
& \leq \int_{0}^{\min \left\{\bar{D}_{1}(\alpha), Y-y\right\}}\left(D_{1}^{-1}(s)-\alpha\right) d s \\
& =\pi_{1}\left(\alpha, \bar{D}_{1}(\alpha), q, y\right),
\end{aligned}
$$

provided that $\min \{x, Y-y\} \neq 0$. Now $x \geq \beta>0$ by definition. It remains to rule out $Y-y=0$. But if $y=Y$, player 2 could increase his profit by bidding $(2 \alpha, Y)$, contradicting the equilibrium property. Hence $y<Y$ has to hold for a best response by player 2. Having dealt with all possibilities, we conclude that $(q-\alpha, x)$ cannot be a best reply for player 1 , for any $x$. Conversely, if $p>2 \alpha$, then player 2's best reply will not have a price component $q=p-\alpha$. As a consequence, in any Nash equilibrium, if $p>2 \alpha$ or $q>2 \alpha$, we must have $p=q$.

If one of the prices, $p$ or $q$, is less than or equal to $2 \alpha$, then the above argument does not go through. We then have either have $p=q$ (equal to $\alpha$ or to $2 \alpha$ ), or we have $(p, q)=(\alpha, 2 \alpha)$ or $(p, q)=(2 \alpha, \alpha)$.

QED 
Corollary 2 Let $(p, x, q, y) \in \mathcal{P} \times \mathcal{Y} \times \mathcal{P} \times \mathcal{Y}$ be a Nash equilibrium in pure strategies. Then

(a) $\bar{D}_{1}(p)+\bar{D}_{2}(q) \geq Y$.

Proof. Suppose $(p, x, q, y)$ is a Nash equilibrium. By corollary 1, there are four cases to be considered.

Case 1, " $p=q=\alpha$." By assumption $\bar{D}_{1}(\alpha)+\bar{D}_{2}(\alpha)>Y$ [see end of section 3].

Case 2, " $p=\alpha, q=2 \alpha$." By assumption, $\bar{D}_{1}(\alpha)+\bar{D}_{2}(2 \alpha)>Y$

Case 3, " $p=2 \alpha, q=\alpha$." By assumption, $\bar{D}_{1}(2 \alpha)+\bar{D}_{2}(\alpha)>Y$.

Case 4, " $p=q>\alpha$." We show that if $\bar{D}_{1}(p)+\bar{D}_{2}(q)<Y$, then player 1 (or, analogously, player 2) has a better reply, contradicting the equilibrium property. $\bar{D}_{1}(p)+\bar{D}_{2}(q)<Y$ and optimal quantity choice implies $x=\bar{D}_{1}(p), y=\bar{D}_{2}(q)$ and $x+y<Y$. Consider the alternative strategy $(\alpha, x)$ for player 1 . Since by assumption $x+y<Y$, we have $Z_{1}(p, x, p, y)=$ $Z_{1}(\alpha, x, p, y)=x$. Thus player 1 receives the same amount but pays less. Hence his payoff is greater. As a consequence, $(p, x)$ cannot be a best reply.

QED

Proposition 3 (pure strategy equilibrium). Let $(p, x, q, y) \in \mathcal{P} \times \mathcal{Y} \times \mathcal{P} \times \mathcal{Y}$ be a pair of pure strategies satisfying $p=q$. Then $(p, x, q, y)$ is a Nash equilibrium point if and only if the following set of conditions is satisfied.

(b) $Z_{1}(p, x, q, y)=\min \left\{\bar{D}_{1}(p), \check{z}_{1}(Y, y)\right\}$, and equivalently for player 2 ,

(c) $\int_{0}^{\min }\left\{\bar{D}_{1}(\alpha), Y-y\right\}\left(D_{1}^{-1}(s)-\alpha\right) d s \leq \pi_{1}(p, x, q, y)$, and equivalently for player 2 ,

(d) $\int_{\min \left\{\bar{D}_{1}(p), \check{z}_{1}(Y, y)\right\}}^{\bar{D}_{1}(p+\alpha)}\left(D_{1}^{-1}(s)-p\right) d s \leq \alpha \bar{D}_{1}(p+\alpha)$, and equivalently for player 2.

Note an interesting difference to MM's theorem. Their condition (ii) guarantees that the market clears. In the finite action auction game, according to condition $(a)$, market clearing provides only an upper bound for the resulting equilibrium price, so there can be excess demand in equilibrium. Condition $(d)$, however, restrains the amount of excess demand that is possible in any pure strategy equilibrium.

Proof. SUFFICIENCY. Suppose $(p, x, p, y)$ satisfies $(b)$ to $(d)$. We have to show that this implies that $(p, x, p, y)$ is a Nash equilibrium. In other words, we must establish that there is no alternative strategy $\left(p^{\prime}, x^{\prime}\right)$ for agent 1 which is a better reply than $(p, x)$, and similarly for player 2 . We consider three kinds of deviations separately.

First, consider a deviation to a strategy $\left(p^{\prime}, x^{\prime}\right)$ such that $p^{\prime}>p$. These strategies have the advantage that player 1 avoids being rationed. The cheapest way to achieve that effect is by out-bidding his rival by the smallest possible price increment, $\alpha$. The preferred quantity to purchase for player 1 at this price is by definition $\bar{D}_{1}(p+\alpha)$. Thus, the best candidate for a better reply is $\left(p+\alpha, \bar{D}_{1}(p+\alpha)\right)$. The payoff is

$$
\begin{array}{rlrl}
\pi_{1}\left(p+\alpha, \bar{D}_{1}(p+\alpha), p, y\right) & =\int_{0}^{\bar{D}_{1}(p+\alpha)}\left(D_{1}^{-1}(s)-(p+\alpha)\right) d s & \\
& \leq \int_{0}^{\min \left\{\bar{D}_{1}(p), \tilde{z}_{1}(Y, y)\right\}}\left(D_{1}^{-1}(s)-p\right) d s & & {[\mathrm{by}(d)]} \\
& =\pi_{1}(p, x, p, y), & & {[\mathrm{by}(b)]}
\end{array}
$$


so $\left(p+\alpha, \bar{D}_{1}(p+\alpha)\right)$ is not a better reply than $(p, x)$.

Next, consider deviations of the form $\left(p, x^{\prime}\right)$ [so the price component is unchanged compared to the original strategy $(p, x)]$. From $(b)$ we know that $Z_{1}(p, x, p, y)$ is equal either to $\bar{D}_{1}(p)$ or to $\check{z}_{1}(Y, y)$, whichever is smaller. Suppose, first, that $Z_{1}(p, x, p, y)=\bar{D}_{1}(p)$. That means that the quantity component $x$ in the strategy leads to the allocation of the preferred quantity, $\bar{D}_{1}(p)$, at price $p$. In this case, clearly, no alternative quantity component $x^{\prime}$ can make things better for player 1 . So suppose, second, that $Z_{1}(p, x, p, y)=\check{z}_{1}(Y, y)<\bar{D}_{1}(p)$. That means that player 1 is rationed. He cannot purchase his preferred quantity at price $p$. The best he can do then is to bid the maximum allowed quantity, $Y$, which allows him to purchase $\check{z}_{1}(Y, y)$ units. Any alternative quantity component $x^{\prime}$ leads to an allocation of at most $\check{z}_{1}(Y, y)$ units, so no $x^{\prime}$ is better than $x$.

Finally, consider deviations $\left(p^{\prime}, x^{\prime}\right)$ with $p^{\prime}<p$. The advantage of these deviations is that player 1 pays less per unit he receives. However, he can get at most the residual demand, i.e. the quantity not demanded by player 2 . The best candidate for a deviation of this sort is $\left(\alpha, \bar{D}_{1}(\alpha)\right)$. This strategy leads to an allocation of $Z_{1}\left(\alpha, \bar{D}_{1}(\alpha), p, y\right)=\min \left\{\bar{D}_{1}(\alpha), Y-y\right\}$ units to player 1 , and his payoff is

$$
\begin{aligned}
\pi_{1}\left(\alpha, \bar{D}_{1}(\alpha), p, y\right) & =\int_{0}^{\min \left\{\bar{D}_{1}(\alpha), Y-y\right\}}\left(D_{1}^{-1}(s)-\alpha\right) d s \\
& \leq \pi_{1}(p, x, p, y),
\end{aligned}
$$

so $\left(\alpha, \bar{D}_{1}(\alpha)\right)$ is not a better response than $(p, x)$.

This establishes that there is no profitable deviation for player 1. Analogous arguments can be made for player 2 , hence $(p, x, p, y)$ is indeed a Nash equilibrium.

NECESSITY. Suppose that $(p, x, p, y)$ is a Nash equilibrium point. We derive conditions $(b),(c)$, and $(d)$ in three steps.

Step 1, " $\forall z \in\left[0, Y^{2} /(Y+y)\right] \cap \mathcal{Y} \exists x \in \mathcal{Y}$ s.t. $Z_{1}(p, x, p, y)=z$, and similarly for player 2." This says that, if both players set the same price, then, for any quantity $z$ on the grid between 0 and $Y^{2} /(Y+y)$, player 1 can always find a quantity bid $x$, such that $z$ is allocated to him. That means that there are no "holes" (besides the grid) in the set of quantities player 1 can purchase.

Let $z \in\left[0, Y^{2} /(Y+y)\right] \cap \mathcal{Y}$. If $z+y \leq Y$, then $x=z$ will do. In case $z+y>Y$, we want to find $x \in \mathcal{Y}$ such that $x+y>Y$ and

$$
z=\check{z}_{1}(x, y)=\max \left\{w \in \mathcal{Y}: w \leq \frac{x}{x+y} Y\right\} .
$$

Checking $x=z$ and $x=Y$ yields

$$
\frac{z}{z+y} Y<z \leq \frac{Y}{Y+y} Y .
$$

Since $x /(x+y)$ is increasing in $x$, there is a smallest $x \in \mathcal{Y}$ with $z \leq \frac{x}{x+y} Y$. As a consequence, $z<x$. Hence $x-Y / \bar{m} \in \mathcal{Y}$ and

$$
\frac{x-Y / \bar{m}}{x-Y / \bar{m}+y} Y<z \leq \frac{x}{x+y} Y .
$$


Next we claim $\left|\frac{x}{x+y} Y-\frac{x-Y / \bar{m}}{x-Y / \bar{m}+y} Y\right|<\beta$. This implies that $z$ is the only grid point in the interval $\left[\frac{x-Y / \bar{m}}{x-Y / \bar{m}+y} Y, \frac{x}{x+y} Y\right]$ and, therefore, $z=\check{z}_{1}(x, y)$. The claim amounts to

$$
\begin{array}{ll}
\frac{x}{x+y}-\frac{x-Y / \bar{m}}{x+y-Y / \bar{m}}<\frac{1}{\bar{m}} & \text { or } \\
\frac{\bar{m} x}{\bar{m} x+\bar{m} y}<\frac{\bar{m} x+x+y-(1+1 / \bar{m}) Y}{\bar{m} x+\bar{m} y-Y} & \text { or } \\
\bar{m} y Y+(x+y) Y<\bar{m}(x+y)(x+y), &
\end{array}
$$

which holds true, since $Y<x+y$ implies $\bar{m} Y y<\bar{m}(x+y) y$ and $Y \leq \bar{m} z<\bar{m} x$ implies $(x+y) Y<\bar{m}(x+y) x$.

Step 2, " $Z_{1}(p, x, p, y)=\check{z}_{1}(x, y)=\min \left\{\bar{D}_{1}(p), \check{z}_{1}(Y, y)\right\}$, and similarly for player 2." It follows from corollary 2 that $x+y \geq Y$. [For otherwise, $x+y<Y \leq \bar{D}_{1}(p)+\bar{D}_{2}(p)$. Without loss of generality, let us pick player 1 and assume that $x<\bar{D}_{1}(p)$ and $x<Y-y$. Then the player could increase his payoff by choosing $x^{\prime}=\min \left\{Y-y, \bar{D}_{1}(p)\right\}$. Hence in equilibrium, $x+y \geq Y$ must hold as asserted.] But $x+y \geq Y$ implies the first equality, $Z_{1}(p, x, p, y)=\check{z}_{1}(x, y)$.

For the second equality, suppose $\bar{D}_{1}(p) \leq \check{z}_{1}(Y, y)$. This implies, by step 1 , that there exists some $x^{\prime}$ such that $Z_{1}\left(p, x^{\prime}, p, y\right)=\bar{D}_{1}(p)$. $\bar{D}_{1}(p)$ is by definition the preferred quantity for player 1 , given price $p$, so any best reply must be such that $Z_{1}\left(p, x^{\prime}, p, y\right)=\bar{D}_{1}(p)$.

Suppose now that $\bar{D}_{1}(p)>\check{z}_{1}(Y, y)$. This means that player 1 cannot receive the quantity he demands at the price $p$. Any strategy with price component $p$ and with a quantity component $x$ such that less then the maximum possible quantity, $\check{z}_{1}(Y, y)$, is allocated to player 1 , can be beaten by the alternative strategy $(p, Y)$. Thus, any best reply with price component $p$ must be such that $Z_{1}(p, x, p, y)=\check{z}_{1}(Y, y)$. [Note that this does not imply $x=Y$.] This proves $(b)$.

Step 3. In what follows, $\left(p^{\prime}, x^{\prime}\right)$ denotes an alternative strategy for player 1 . We derive the implications of the fact that in equilibrium, there cannot be such an alternative strategy that is a better reply for player 1 against $(p, y)$ than $(p, x)$.

Consider first alternative strategies with $p^{\prime}<p$. In this case, player 2 will not be rationed, and player 1 will receive either the amount not purchased by player $2, Y-y$, or his bid, $x^{\prime}$, whichever is smaller. Clearly, the best candidate for an alternative move of this kind is to bid the lowest possible price, $\alpha$, and the demand at this low price, $\bar{D}_{1}(\alpha)$. We have

$$
\pi_{1}\left(\alpha, \bar{D}_{1}(\alpha), p, y\right)=\int_{0}^{\min \left\{\bar{D}_{1}(\alpha), Y-y\right\}}\left(D_{1}^{-1}(s)-\alpha\right) d s .
$$

This cannot be greater than $\pi_{1}(p, x, p, y)$ [otherwise $(p, x)$ would not be a best reply], which implies $(c)$.

Consider now deviations with $p^{\prime}>p$. In this case player 1 will not be rationed. Clearly, the best candidate for such an alternative move is $\left(p+\alpha, \bar{D}_{1}(p+\alpha)\right)$. If this move is not better than the original one, then there is no alternative move with $p^{\prime}>p$ that could beat $(p, x)$. The payoff is

$$
\pi_{1}\left(p+\alpha, \bar{D}_{1}(p+\alpha), p, y\right)=\int_{0}^{\bar{D}_{1}(p+\alpha)}\left(D_{1}^{-1}(s)-(p+\alpha)\right) d s
$$


Because $(p, x)$ is a best reply, this term cannot be greater than $\pi_{1}(p, x, p, y)$. Combined with step 2, this implies $(d)$.

QED

\section{$6 \quad$ Asymptotic properties}

Intuitively, a finer grid moves the finite action model closer to the continuous action model. In this section we investigate in which sense this intuition is confirmed.

According to corollary 1, the finite action game has two types of equilibria. One type involves the same price bids by both players $(p=q$, as in the continuous action game); the other type involves unequal and close to zero price bids by both bidders. Subject to some additional restriction on the demand functions, proposition 4 shows that this latter type of equilibrium ceases to exist if the grid is fine enough. From corollary 2 we know that there can be excess demand in equilibrium. Proposition 5 implies that equilibrium excess demand vanishes if the price grid is fine enough compared to the quantity grid, and if both grids become arbitrarily fine. Proposition 6 establishes that there is an equivalence relationship between the equilibria of the continuous action game and the $\epsilon$-equilibria of the finite action game, with $\epsilon$ depending on the fineness of the grid.

Proposition 4. Suppose $D_{1}(0)>Y / 2$ and $D_{2}(0)>Y / 2$. Then, for small enough $\alpha$ and $\beta$, $p=q$ in any pure strategy equilibrium.

Before moving on to the proof, observe that, if $(\alpha, x, 2 \alpha, y) \in \mathcal{P} \times \mathcal{Y} \times \mathcal{P} \times \mathcal{Y}$ is a Nash equilibrium, then $Z_{2}(\alpha, x, 2 \alpha, y)>Y / 2$. For otherwise, by step 1 in the previous proof, player 2 could acquire the same quantity at a lower price, contradicting the equilibrium property. Of course, $Z_{2}(\alpha, x, 2 \alpha, y) \leq \bar{D}_{2}(2 \alpha)$. Therefore, the additional requirement made in this proposition that $D_{1}(0)>Y / 2$ and $D_{2}(0)>Y / 2$ adds less restriction to the model than one might think at first glance.

Proof. Fix some $\epsilon \in(0,1)$ with $D_{i}(\epsilon)>Y / 2$, for $i=1,2$, and choose some $E>0$ such that $D_{i}(\epsilon)>Y / 2+4 E$ for $i=1,2$ and $Y>Y / 2+4 E$. Set $\alpha^{0}=(E \epsilon) /(2 Y)>0$ and $\beta^{0}=E / 3>0$. This implies that $\alpha<(E \epsilon) / Y, 2 \beta<E$, and $\bar{D}_{i}(\alpha) \geq \bar{D}_{i}(2 \alpha)>Y / 2+3 E$, for all $\alpha \leq \alpha^{0}, \beta \leq \beta^{0}$, $i=1,2$. Let us proceed with sufficiently small $\alpha$ and $\beta$, i.e. $\alpha \leq \alpha^{0}$ and $\beta \leq \beta^{0}$. Given the prices $p=\alpha$ and $q=2 \alpha$, maximization of her payoff with respect to quantity has player 2 bid $y=\bar{D}_{2}(2 \alpha)$ so that player 1 receives $Z_{1}(\alpha, x, 2 \alpha, y)<Y / 2-3 E$. Now consider the alternative strategy $\left(2 \alpha, x^{\prime}\right)$ for player 1 where $x^{\prime}$ is chosen so that $Z_{1}\left(2 \alpha, x^{\prime}, 2 \alpha, y\right)=Z_{1}(\alpha, x, 2 \alpha, y)+k \beta$ and $k$ is maximal with $k \beta \leq 3 E$. Then $k \beta \geq 2 E$. When making this change, player 1 gains at least $2 E \cdot D_{1}^{-1}\left(Z_{1}\left(2 \alpha, x^{\prime}, 2 \alpha, y\right)\right)$ and loses at most $2 \alpha Y$. Next $Z_{1}\left(2 \alpha, x^{\prime}, 2 \alpha, y\right) \leq Y / 2<D_{1}(\epsilon)$. Hence $D_{1}^{-1}\left(Z_{1}\left(2 \alpha, x^{\prime}, 2 \alpha, y\right)\right)>D_{1}^{-1}\left(D_{1}(\epsilon)\right)=\epsilon$. Hence the gains are at least $2 E \epsilon$ while the losses are at most $2 \alpha Y$. Since $\alpha$ is sufficiently small, the gains exceed the losses, and $(p, q)=(\alpha, 2 \alpha)$ cannot be part of an equilibrium.

QED

For the next proposition, the price grid ought to be fine enough relative to the quantity grid so that the cost of raising the price by one unit is small compared with the gain from receiving a larger quantity. Given any $\alpha$ and $\beta$ with $D_{1}(0)>2 \beta$ and $D_{2}(0)>2 \beta$, we shall say 
that $\alpha$ is small enough relative to $\beta$, if $\alpha<\alpha^{0}(\beta)$ where $\alpha^{0}(\beta)$ is defined as follows: For $p \in\left[0, D_{i}^{-1}(2 \beta)\right]$, define the strictly positive and continuous function

$$
\Delta_{i}(\beta, p):=\int_{D_{i}(p)-2 \beta}^{D_{i}(p)-\beta}\left(D_{i}^{-1}(s)-p\right) d s .
$$

On the compact interval $\left[0, D_{i}^{-1}(2 \beta)\right], \Delta_{i}(\beta, \cdot)$ assumes a minimum $\Delta_{i}^{*}(\beta)>0$. Set $\Delta^{0}=\min \left\{\Delta_{1}^{*}, \Delta_{2}^{*}\right\}$ and $\alpha^{0}=\Delta^{0} / Y$.

Proposition 5. Suppose $D_{1}(0)>2 \beta, D_{2}(0)>2 \beta$ and $\alpha$ small enough relative to $\beta$. If $(p, x, p, y) \in \mathcal{P} \times \mathcal{Y} \times \mathcal{P} \times \mathcal{Y}$ is an equilibrium, then $\bar{D}_{1}(p)+\bar{D}_{2}(p) \leq Y+6 \beta$, i. e. excess demand is at most $6 \beta$.

An immediate corollary of this is that if both components of the grid, $\alpha$ and $\beta$, converge to zero and $\alpha$ converges sufficiently fast relative to $\beta$, then equilibrium excess demand eventually vanishes.

Proof. For $\bar{m}=2, \ldots, 6$, the assertion is trivially true. From here on assume $\bar{m} \geq 7$ such that $D_{1}(0)>2 \beta$ and $D_{2}(0)>2 \beta$. Also assume $\alpha<\alpha^{0}(\beta)$.

Next let $(p, x, p, y)$ be a Nash equilibrium of the finite game associated with $\alpha$ and $\beta$. We start with some useful observations:

1. $p<\bar{p}$.

2. If $(p, x, p, y)$ is a Nash equilibrium and $\bar{D}_{1}(p)>\check{z}_{1}(x, y)+3 \beta$, then $x=Y$ and $Z_{1}(p, x, p, y)=\check{z}_{1}(Y, y) \geq Y / 2 \geq 2 \beta$.

3. $\check{z}_{1}(x, y)+\check{z}_{2}(x, y) \leq Y$.

One obtains that if $\bar{D}_{i}(p) \leq \check{z}_{i}(x, y)+3 \beta$ for both $i$, then $\bar{D}_{1}(p)+\bar{D}_{2}(p) \leq Y+6 \beta$. We are done, if we can show that $\bar{D}_{i}(p)>\check{z}_{i}(x, y)+3 \beta$ cannot occur. Without loss of generality, suppose $\bar{D}_{1}(p)>\check{z}_{1}(x, y)+3 \beta$. We claim that then by placing the alternative bid $\left(p+\alpha, \bar{D}_{1}(p)\right)$, investor 1 could get $\pi_{1}\left(p+\alpha, \bar{D}_{1}(p), p, y\right)>\pi_{1}(p, x, p, y)$, contradicting the hypothesis that $(p, x, p, y)$ is a Nash equilibrium. Therefore, to the contrary $\bar{D}_{1}(p) \leq \check{z}_{1}(x, y)+3 \beta$ has to hold. To show the claim, we distinguish two cases.

Case 1, $\left|\bar{D}_{1}(p)-D_{1}(p)\right|<\beta$. Then $D_{1}(p)>\check{z}_{1}(x, y)+2 \beta$. Further

$$
\begin{aligned}
& \pi_{1}\left(p+\alpha, \bar{D}_{1}(p), p, y\right)=\int_{0}^{\bar{D}_{1}(p)}\left(D_{1}^{-1}(s)-(p+\alpha)\right) d s \\
= & \int_{0}^{\bar{D}_{1}(p)}\left(D_{1}^{-1}(s)-p\right) d s-\alpha \bar{D}_{1}(p) \\
= & \int_{0}^{\check{z}_{1}(x, y)}\left(D_{1}^{-1}(s)-p\right) d s+\int_{\check{z}_{1}(x, y)}^{\bar{D}_{1}(p)}\left(D_{1}^{-1}(s)-p\right) d s-\alpha \bar{D}_{1}(p) \\
\geq & \pi_{1}(p, x, p, y)+\int_{D_{1}(p)-2 \beta}^{D_{1}(p)-\beta}\left(D_{1}^{-1}(s)-p\right) d s-\alpha \bar{D}_{1}(p) \\
\geq & \pi_{1}(p, x, p, y)+\Delta^{0}(\beta)-\alpha \bar{D}_{1}(p) \\
\geq & \pi_{1}(p, x, p, y)+\left[\alpha^{0}(\beta)-\alpha\right] Y>\pi_{1}(p, x, p, y) .
\end{aligned}
$$


Case 2, $\left|\bar{D}_{1}(p)-D_{1}(p)\right| \geq \beta$. In this case, $\bar{D}_{1}(p)=Y$ and $D_{1}(p) \geq Y+\beta$. There exists $p^{\prime} \in\left(p, D_{1}^{-1}(2 \beta)\right)$ with $D_{1}\left(p^{\prime}\right)=\bar{D}_{1}(p)$ and, consequently,

$$
\begin{aligned}
& \int_{\check{z}_{1}(x, y)}^{\bar{D}_{1}(p)}\left(D_{1}^{-1}(s)-p\right) d s>\int_{\check{z}_{1}(x, y)}^{D_{1}\left(p^{\prime}\right)}\left(D_{1}^{-1}(s)-p^{\prime}\right) d s \\
> & \int_{D_{1}\left(p^{\prime}\right)-2 \beta}^{D_{1}\left(p^{\prime}\right)-\beta}\left(D_{1}^{-1}(s)-p^{\prime}\right) d s \geq \Delta^{0}(\beta) .
\end{aligned}
$$

The remainder of the argument is the same as in case 1.

For equilibria of the form $(p, x, p, y) \in S \times S$, further asymptotic results can be obtained by letting the grid lengths go to zero. In accordance with the rationale of our finite action model, such a result is only of interest if $(p, x, p, y)$ lies on all the grids involved.

Proposition 6. Consider two strictly increasing sequences $\bar{n}_{k}, k \in \mathbb{N}$, and $\bar{m}_{k}, k \in \mathbb{N}$, in $\mathbb{N} \backslash\{1\}$. Let $\mathcal{P}_{k}, \mathcal{Y}_{k}$, and $\mathcal{S}_{k}$ denote the corresponding sequences of grids and $\alpha_{k}$ and $\beta_{k}$ denote the corresponding sequences of grid lengths. Set $\epsilon_{k}=3 \beta_{k} \cdot \bar{p}$. Suppose that $(p, x, p, y) \in S \times S$ is a point that belongs to $\mathcal{S}_{k} \times \mathcal{S}_{k}$ for all $k$.

(A) If $(p, x, p, y)$ is a Nash equilibrium of the continuous action game, then it is (for each $k$ ) an $\epsilon_{k}$-equilibrium of the respective finite action game.

(B) If $(p, x, p, y)$ is not a Nash equilibrium of the continuous action game, then it is not (for large $k$ ) an $\epsilon_{k}$-equilibrium of the respective finite action game.

Proof. (A) It suffices to provide the argument for player 1. An analogue holds for player 2. If $(p, x, p, y)$ is a Nash equilibrium of the continuous action game, then $\pi_{1}^{*}(p, x, p, y) \geq \pi_{1}^{*}\left(p^{\prime}, x^{\prime}, p, y\right)$ for all $\left(p^{\prime}, x^{\prime}\right) \in S$. Fix $k \in \mathbb{N}$. The above inequalities imply $\pi_{1}^{*}(p, x, p, y) \geq \pi_{1}^{*}\left(p^{\prime}, x^{\prime}, p, y\right)$ for all $\left(p^{\prime}, x^{\prime}\right) \in \mathcal{S}_{k}$. Now observe that for all $\left(p^{\prime \prime}, x^{\prime \prime}, q^{\prime \prime}, y^{\prime \prime}\right) \in \mathcal{S}_{k} \times \mathcal{S}_{k}, Z_{1}\left(p^{\prime \prime}, x^{\prime \prime}, q^{\prime \prime}, y^{\prime \prime}\right) \leq$ $Z_{1}^{*}\left(p^{\prime \prime}, x^{\prime \prime}, q^{\prime \prime}, y^{\prime \prime}\right)$, with the difference at most $\beta_{k}$. In the payoff formula $(1), p^{\prime \prime} \in[0, \bar{p}]$ and $D_{1}^{-1}(s) \in[0, \bar{p}]$ and therefore $\left|D_{1}^{-1}(s)-p^{\prime \prime}\right| \leq \bar{p}$. Hence

$$
\begin{aligned}
\left|\pi_{1}^{*}\left(p^{\prime \prime}, x^{\prime \prime}, q^{\prime \prime}, y^{\prime \prime}\right)-\pi_{1}\left(p^{\prime \prime}, x^{\prime \prime}, q^{\prime \prime}, y^{\prime \prime}\right)\right| & =\left|\int_{Z_{1}\left(p^{\prime \prime}, x^{\prime \prime}, q^{\prime \prime}, y^{\prime \prime}\right)}^{Z_{1}^{*}\left(p^{\prime \prime}, x^{\prime \prime}, q^{\prime \prime}, y^{\prime \prime}\right)}\left(D_{1}^{-1}(s)-p^{\prime \prime}\right) d s\right| \\
& \leq \int_{Z_{1}\left(p^{\prime \prime}, x^{\prime \prime}, q^{\prime \prime}, y^{\prime \prime}\right)}^{Z_{1}^{*}\left(p^{\prime \prime}, x^{\prime \prime}, q^{\prime \prime}, y^{\prime \prime}\right)}\left|D_{1}^{-1}(s)-p^{\prime \prime}\right| d s \\
& \leq \int_{Z_{1}\left(p^{\prime \prime}, x^{\prime \prime}, q^{\prime \prime}, y^{\prime \prime}\right)}^{Z_{1}^{*}\left(p^{\prime \prime}, x^{\prime \prime}, q^{\prime \prime}, y^{\prime \prime}\right)} \bar{p} d s \\
& =\left[Z_{1}^{*}\left(p^{\prime \prime}, x^{\prime \prime}, q^{\prime \prime}, y^{\prime \prime}\right)-Z_{1}\left(p^{\prime \prime}, x^{\prime \prime}, q^{\prime \prime}, y^{\prime \prime}\right)\right] \cdot \bar{p} \\
& \leq \beta_{k} \cdot \bar{p}=\frac{1}{3} \epsilon_{k} .
\end{aligned}
$$

Since this inequality applies to $(p, x, p, y),\left(p^{\prime}, x^{\prime}, p, y\right) \in \mathcal{S}_{k} \times \mathcal{S}_{k}$, the $\epsilon_{k}$-equilibrium condition follows: $\pi_{1}(p, x, p, y) \geq \pi_{1}\left(p^{\prime}, x^{\prime}, p, y\right)-\frac{1}{3} \epsilon_{k} \geq \pi_{1}\left(p^{\prime}, x^{\prime}, p, y\right)-\epsilon_{k}$ for all $\left(p^{\prime}, x^{\prime}\right) \in \mathcal{S}_{k}$. 
(B) If $(p, x, q, y)$ is not a Nash equilibrium of the continuous action game, let us assume without loss of generality that player 1 gains from deviation. Then there exists $\left(p^{\prime}, x^{\prime}\right) \in S$ with $\pi_{1}^{*}(p, x, p, y)<\pi_{1}^{*}\left(p^{\prime}, x^{\prime}, p, y\right)$. Let us fix such a strategy $\left(p^{\prime}, x^{\prime}\right)$ and choose $\delta>0$ such that $\pi_{1}^{*}\left(p^{\prime}, x^{\prime}, p, y\right)-\pi_{1}^{*}(p, x, q, y)>5 \delta$.

Case $p^{\prime}=p$. For each $k$, set $x_{k}=\min \left\{z \in \mathcal{Y}_{k}: x^{\prime} \leq z\right\}$. Observe that the function $\tilde{Z}_{1}(X) \equiv Z_{1}^{*}(p, X, p, y)$ is continuous in $X$ and $x_{k} \rightarrow x^{\prime}$ as $k \rightarrow \infty$. Hence for sufficiently large $k,\left|Z_{1}^{*}\left(p, x_{k}, p, y\right)-Z_{1}^{*}\left(p, x^{\prime}, p, y\right)\right|<\delta / \bar{p}$ and, by variation of an argument used in the proof of $(\mathrm{A})$,

$$
\left|\pi_{1}^{*}\left(p, x_{k}, p, y\right)-\pi_{1}^{*}\left(p, x^{\prime}, p, x\right)\right|<\delta
$$

Based on the proof of $(\mathrm{A})$, we conclude further that

$\left|\pi_{1}^{*}(p, x, p, y)-\pi_{1}(p, x, p, y)\right|<\epsilon_{k}$ and

$\left|\pi_{1}^{*}\left(p, x_{k}, p, y\right)-\pi_{1}\left(p, x_{k}, p, y\right)\right|<\epsilon_{k}$.

For sufficiently large $k$, we obtain $\epsilon_{k}<\delta$ and, by repeated application of the triangle inequality, $\pi_{1}\left(p, x_{k}, p, y\right)>\pi_{1}(p, x, p, y)+2 \delta>\pi_{1}(p, x, p, y)+\epsilon_{k}$ or $\pi_{1}(p, x, p, y)<\pi_{1}\left(p, x_{k}, p, y\right)-\epsilon_{k}$. This shows that $(p, x, p, y)$ is not an $\epsilon_{k}$-equilibrium.

Case $p^{\prime}>p$. For each $k$, set $x_{k}=\min \left\{z \in \mathcal{Y}_{k}: x^{\prime} \leq z\right\}$ and $p_{k}=\min \left\{q \in \mathcal{P}_{k}: p^{\prime} \leq q\right\}$. For sufficiently large $k, p_{k}>p$. Since $\left|p_{k}-p^{\prime}\right| \leq \alpha$, it follows

$$
\left|\pi_{1}^{*}\left(p^{\prime}, x^{\prime}, p, y\right)-\pi_{1}^{*}\left(p_{k}, x^{\prime}, p, y\right)\right|<\alpha_{k} Y .
$$

Similar reasoning as in the previous case yields for sufficiently large $k: \epsilon_{k}<\delta, \alpha_{k} Y<\delta$ and

$\left|\pi_{1}^{*}\left(p_{k}, x_{k}, p, y\right)-\pi_{1}^{*}\left(p_{k}, x^{\prime}, p, x\right)\right|<\delta$

$\left|\pi_{1}^{*}(p, x, p, y)-\pi_{1}(p, x, p, y)\right|<\epsilon_{k}$

$\left|\pi_{1}^{*}\left(p_{k}, x_{k}, p, y\right)-\pi_{1}\left(p_{k}, x_{k}, p, y\right)\right|<\epsilon_{k}$.

Hence, for sufficiently large $k$, repeated application of the triangle inequality yields $\pi_{1}\left(p_{k}, x_{k}, p, y\right)>\pi_{1}(p, x, p, y)+\delta>\pi_{1}(p, x, p, y)+\epsilon_{k}$

which implies that $(p, x, p, y)$ is not an $\epsilon_{k}$-equilibrium.

Case $p^{\prime}<p$. Use the same logic as for the case $p^{\prime}>p$.

\section{Conclusion}

The problem of non-existence of an equilibrium in the discriminatory price auction can be overcome by resorting to the realistic assumption of smallest bid increments. The resulting finite action game shares several features of the continuous action game. For a meaningful comparison, a uniform price auction should also be modelled as a finite action game, with the same price and quantity grids as the discriminatory price auction. Our analysis suggests that this can be done. 


\section{References}

Chari, V. V. and Weber, R. J. (1992) "How the U.S. Treasury Should Auction its Debt," Federal Reserve Bank of Minneapolis Quarterly Review 16(4), 3-12.

Friedman, M. (1960) A Program for Monetary Stability, New York: Fordham University Press.

Menezes, F. M. and Monteiro, P. K. (1995) "Existence of equilibrium in a discriminatory price auction," Mathematical Social Sciences 30, 285-292.

Nash, J. (1950) "Equilibrium Points in n-Person Games," Proceeding of the National Academy of Sciences of the U.S.A. 36, 48-49.

Nautz, D. (1995) "Zur Feinsteuerung des Geldmarktes durch die Wertpapierpensionsgeschäfte der Bundesbank," Zeitschrift für Wirtschafts- und Sozialwissenschaften 115(4), 623-644.

Nyborg, K. G. and Sundaresan, S. (1996) "Discriminatory versus Uniform Treasury Auctions: Evidence from When-Issued Transactions," Journal of Financial Economics 42(1), 63-104. U.S. Treasury (1997) "Treasury bill, note \& bond tender instructions," Department of the Treasury, Bureau of the Public Debt, PD F 5382(I), available from ftp://ftp.publicdebt. treas.gov/pdf5382.pdf, uploaded on 09/04/97. 